\title{
The influences of dietary inputs on the neonatal gastrointestinal tract : Managing the development of a complex ecosystem
}

\author{
R.K. Buddington \\ Department of Biological Sciences, \\ Mississippi State University \\ Mississippi State, MS 39762-5759, USA
}

\begin{abstract}
The gastrointestinal tract can be considered as a small, but complex, ecosystem that includes abiotic (physical and chemical characteristics) and biotic (resident microbiota) components. Many of the ecological principles known for river systems are shared by the GIT and are useful for understanding the influences of dietary inputs. Although each species has a genetically programmed sequence of postnatal changes in GIT structure, functions, and the resident microbiota, the patterns can be manipulated by dietary inputs. Important questions remain to be answered before dietary inputs can be effectively used to "manage" postnatal development of the GIT and thereby optimize health and development of animals.
\end{abstract}

KEY WORDS: gastrointestinal tract, bacteria, mucosa, probiotics, prebiotics, synbiotics

\section{INTRODUCTION}

The gastrointestinal tract varies more in structure than any other vertebrate organ system (Stevens, 1988). Genetic determinants, which are selected during evolution, match GIT structure and functions to the composition of the natural diet, whether this be milk or solid food. Despite the variation, the GIT has certain characteristics that are shared by most vertebrates. Specifically, dietary inputs are first stored in a reservoir, such as the stomach of mammals and crop of birds. After initial processing, the digesta enter the small intestine and proceed distally, and are eventually eliminated. 
The GIT is comparable to many river systems that originate from reservoirs that collect drainage from watersheds. Movement of food from the stomach into the intestine is regulated by the pyloric sphincter, just as the release of water into the river can be regulated by the dam. Water velocity is usually fast below the reservoir and gradually slows, similar to the transit of food through the small and large intestine. In light of the similarities, many of the ecological principles applied to river systems can be used to better understand the complex interactions between dietary inputs, GIT structure and functions, and the resident bacteria. One critical consideration is that management approaches must be modificd to meet the specific characteristics of different ecosystems, be they the GIT of different species or life history stages, or different river systems.

Instead of providing a comprehensive assessment of the literature, this contribution largely reviews the different approaches that are presented by the contributors to this symposium and are currently in use or being examined as potential "management tools" to optimize development of the GIT. Before doing so, I summarize in the first section the age, region, and species differences in GIT structure, functions, and the resident microbiota. For illustrative purposes I present parallels with applicable characteristics of river systems. A subsequent section describes the different management approaches that can be used to influence the developing GIT. The concluding section present perspectives about postnatal management of the gastrointestinal ecosystem.

\section{GIT CHARACTERISTICS: SPATIAL, TEMPORAL, AND SPECIES DIFFERENCES}

The variation in vertebrate GIT structure and functions correponds with the wide diversity of natural diets (Stevens, 1988). There are also age-related changes in GIT characteristics. These are best known for mammals that after birth first drink milk and eventually wean to the adult diet (Buddington, 1994). The best studied examples involve the shift in digestive enzymes, particularly the postnatal decline in lactase and increase in maltase and sucrase. Other important changes include the postnatal onset of gastric secretion (Sangild et al., 1992), shifts in the types, abundance, and distribution of nutrient transporters (Buddington and Diamond, 1989), and changes in the composition and volume of pancreatic and biliary secretions (Pierzynowski et al., 1995). These changes, in combination with changes in dictary input, influence the chemical characteristics in the different regions of the GIT.

There are also changes in the physical characteristics of the different GIT regions during development. Mucosal architecture, which provides a substrate for bacterial attachment and metabolism, undergoes postnatal changes. The most dra- 
matic occur at weaning when there is a decline in villus height, an increase in crypt depth, and changes in the biochemical characteristics of the enterocyte membrane.

Some of the most dramatic postnatal changes involve the GIT microbiota.

The GIT is sterile in utero and after birth is rapidly colonized by bacteria. The principal source of the initial inoculum for mammals is thought to maternal faecal matter and vaginal contaminants. Other environmental sources, such as the nipples of the mother, are important, but even more so for children and animals that are delivered by caesarian section. After the initial colonization of the GIT, the microbiota undergoes a series of changes that are influenced by the introduction of additional species, dietary inputs, and changes in GIT structure and functions. Another important consideration is the modification of the environment by the bacteria, which can facilitate the development of a bacterial assemblage with different qualitative characteristics. Eventually the adult bacterial assemblage develops (Conway, 1996) along with the characteristic regional differences. The sequence of events that culminate in the adult GIT microbiota can take more than two years in humans (Gibson and Roberfroid, 1995) and are similar to the well known principles of "successional ecology".

\section{MANAGING DEVELOPMENT OF THE GIT}

River systems can be managed by alterring the physical and chemical conditions or by changing the composition and/or abundance of the resident biota. The likelihood of attaining a desirable ecosystem is increased if management can begin when a river is "born", not after it is mature. The GIT of newborn animals provides just such an opportunity for management.

There are three obvious benefits from optimizing GIT development:

1. Faster growth, and higher productivity, 2. increased efficiency of diet utilization, and 3. improved health and resistance to disease. This can be accomplished by managing the physical and chemical characteristics of the various GIT regions (i.e., habitats) or by managing the species resident in the different regions. The following reviews information and insights provided by contributors to this symposium about managing the GIT ecosystem during development and includes pertinent additional references.

Managing the developing microbiota. The quantitative and qualitative characteristics of the bacteria resident in the GIT are known to be related to host health. Lactic acid bacteria (e.g., bifidobacteria and lactobacillus) are considered to provide a variety of health benefits (reviewed by Gibson and Roberfroid, 1995; Kelly, 1998). In contrast, certain strains of $E$. coli and species of clostridia, salmonella, and other gram positive bacteria are known pathogens. In addition, the metabolic 
activities of some bacteria can produce substances that are detrimental to the host. In light of the contrasting health implications, there is interest in fostering a resident bacterial assemblage that will promote health and decrease the risk of disease. There are four basic approaches that can be used individually or in combination to modify the composition, hence metabolic activities, of the GIT bacteria. Comparable strategies are used for managing rivers and other ecosystems.

1. Selective removal of target species. Historically, dietary antibiotics have been the principal dietary tool used to "manage" the composition of the GIT bacteria, and the benefits are well established (reviewed by Taljanski-Zygmunt et al., 1998). In a similar manner, pesticides are used to remove unwanted organisms from other ccosystems. The responses to dietary antibiotics are profound during suckling and weaning when the GIT bacteria undergoes dramatic shifts in composition and there is an increase in bacterial-related morbidity and mortality. Of particular benefit is the selective nature of some antibiotics that allow for the removal of target bacteria and do not adversely affect those considered to be beneficial. However, less selective, less costly antibiotics tend be added to diets as prophylactic agents. Uncontrolled use of such antibiotics has raised concerns about the development antibiotic resistant strains of bacteria. The concerns are valid, are already being realized, and have led to use restrictions (reviewed by Taljanski-Zygmunt et al., 1998). This is comparable to the development of resistance by organisms to commonly used pesticides. A less known consequence of some antibiotics is that they can initiate or increase the severity of diarrhoea by disturbing motility and osmoregulatory functions of the colon (Goldhill et al., 1995).

Certain lectins can be considered as alternatives to antibiotics in that they selectively bind to certain bacteria (Kelly, 1998). By doing so, they inhibit attachment to the mucosa and colonization of the GIT. The monosaccharide mannose similarly binds to membrane receptors of some bacteria preventing them from adhering and establishing in the GIT (Oyofo et al., $1989 \mathrm{a}, \mathrm{b}$ ). The milk produced by humans and other mammals contains a diverse array of oligosaccharides, some of which selectively bind to certain species of bacteria, associated toxins, and epithelian cell receptors and may thereby confer health benefits (Newburg, 1997).

2. Alterring the chemical environment. In light of the increasing concerns and restrictions about the use of antibiotics, there has been interest in developing alternative strategies that will adventitiously influence the GIT bacteria. Dietary organic acids, which are bacterial metabolites, and their salts have been shown to be effective at improving growth and health at the time of weaning (reviewed by Roth and Kirchgessner, 1998). Apparently, by alterring the chemical environment, mono-, di- and tricarboxylic acids can inhibit the growth of some bacteria, notably many pathogens. The effect is not attributed to a simple acidification, but involves other mechanisms of action that have not yet been completely understood (Russell and Diez-Gonzalez, 1998). The benefit is that advantageous bacteria, such as the 
lactic acid forms, are affected to a lesser degree, if at all, and as a result increase in relative abundance.

In other ecosystems, changes in the environment caused by one set of organisms encourage the appearance and proliferation of others. This process of facilitation is also known to occur in the GIT. Metabolism by the first bacteria that colonize the GIT lower oxygen tensions and increase redox potential, and by doing so alter the chemical environment such that it is more suitable for the proliferation of the anaerobes that eventually supplant the aerobic forms. Organic acids may accelerate this process, and by doing so enhance the transition to adult GIT bacterial populations that are better adapted to the solid diet than those present during suckling.

3. Using nutrients to manipulate the GIT bacteria. An effective strategy is to provide in the diet substrates that are preferentially utilized by desirable forms of bacteria. The term prebiotics has been used to describe this approach to manage the GIT bacteria. Certain oligosaccharides selectively encourage the growth of some, but not all bacteria. The best studied prebiotic is oligofructose (fructooligosaccharide), and it is known to increase the densities of bifidobacteria in faecal samples of humans and lower the activities of some reductive enzymes implicated in production of carcinogens (Buddington et al., 1996). Several other oligosaccharides are now considered as "bifidogenic factors" (Gibson and Roberfroid, 1995). In addition to already established benefits, the addition of oligofructose and inulin to diets fed to mice reduced mortality caused by Listeria monocytogenes and intestinal densities of Candida albicans (our unpublished data), thereby demonstrating immune stimulation.

Human infants that are breast fed have higher densities of bifidobacteria than those that are fed formula (Gothefors, 1989). Prebiotics, particularly bifidogenic factors, have promise as components of milk replacers in that they may alter the GIT bacteria to make it more comparable to that of breast fed infants. Suckling pigs fed milk replacer containing oligofructose also have more bifidobacteria, with the responses more profound in the small intestine compared to the distal colon (Figure 1). Furthermore, the addition of oligofructose to an oral electrolyte solution accelerates recovery of the GIT bacteria after it has been disturbed by acute diarrhoea (Oli et al., 1998). Therefore, oligosaccharides and other bifidogenic factors can be used as selective "fertilizers" in that they encourage the proliferation of certain bacteria and are associated with a reduction in the relative abundance of other species. However, like different fertilizers, probiotics can elicit different growth responses among species of bacteria (Howard et al., 1995) and yield different proportions of short chain fatty acids (Sunvold et al., 1995), which will have differential effects on the GIT.

An alternative, but related approach, to prebiotics, is to reduce the concentrations of dietary components that are needed by undesireable bacteria. For exam- 


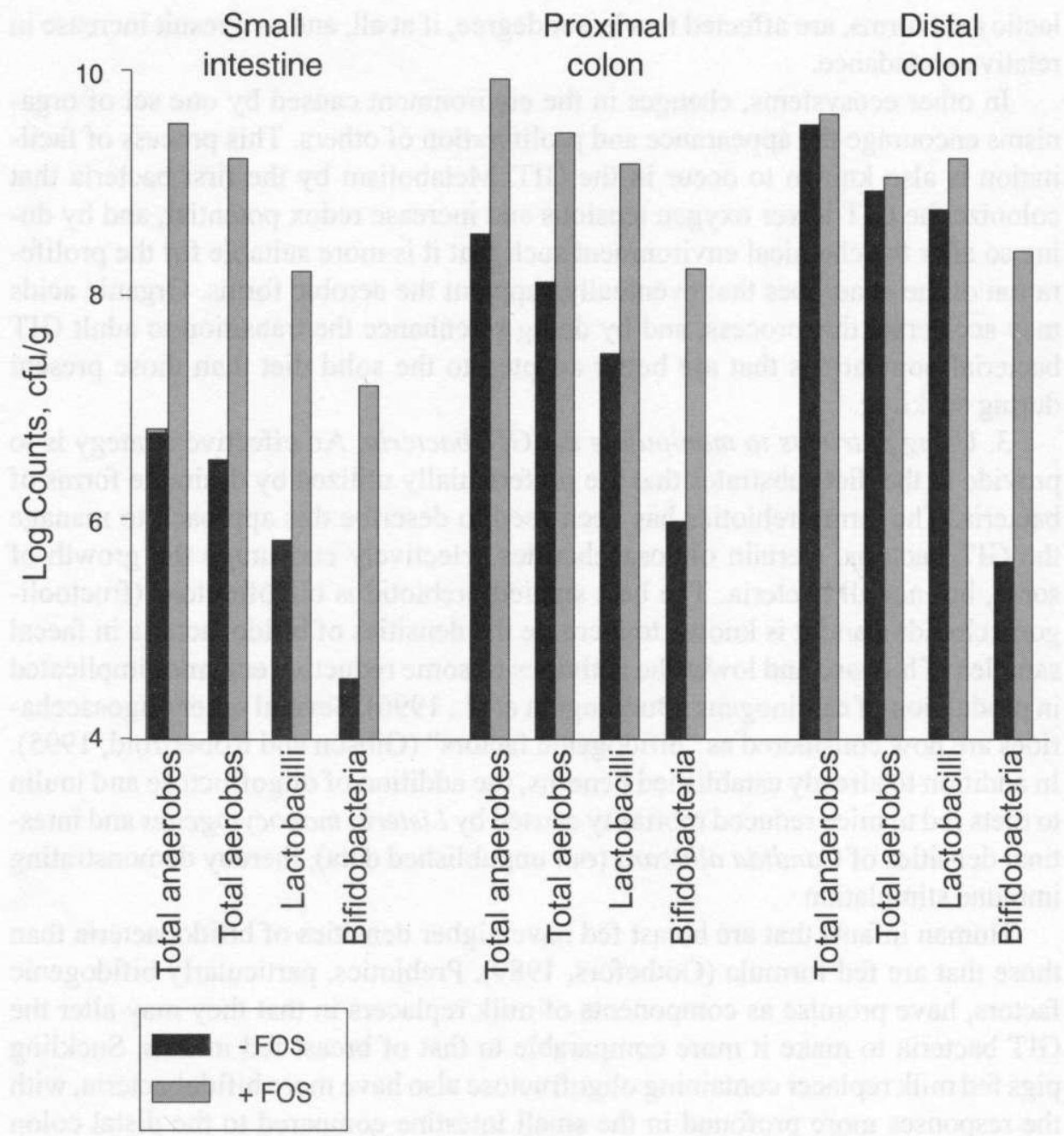

Figure 1. Densities of different bacterial groups in the small intestine, proximal colon, distal colon of suckling pigs fed milk replacers with and without $0.5 \%$ oligofructose (our unpublished data)

ple, infant formulas are often prepared with low iron levels as this reduces the growth of certain pathogens and is thought to lower the risk of enteric-based disease (Balmer et al., 1989).

4. Introduction of desired species. The introduction of beneficial bacteria into the mature GIT (probiotics) has proven successful at improving health (reviewed by Kelly, 1998). However, the introduced species are transient residents of the GIT and can be recovered for only as long as they are included in the diet. There is evidence that the bacteria normally present in the GIT have coevolved with the 
host. For example, the binding of bifidobacteria to enterocytes is species specific (Bernet et al., 1993), and this is probably true for other bacteria. As a consequence introduced species may not be able to successfully compete for limited resources (attachment sites and nutrients) with the bacteria that are already established in the GIT and therefore adapted to the environments in which they reside. This principle of competitive exclusion is well known for other ecosystems.

It is possible to associate the GIT of one species with the microflora from another species (Hentges et al., 1992), but only if the bacteria are introduced into a sterile GIT.

These findings indicate that probiotics can successfully colonize the GIT, but the likelihood is much greater if they are introduced immediately after birth. Careful consideration must be given when selecting the species or strains of bacteria to be included in a probiotic. Effective probiotics will include species that have characteristics that allow them to survive and proliferate in the GIT of the target species. Even so, the natural genotypic diversity between individuals of a species and even between life history stages is sufficient to limit the effectiveness of some probiotics. This would include variation in the carbohydrate residues of the membrane proteins, which can affect bacterial adherence, hence virulence (Kelly, 1998).

\section{MANAGEMENT OF GIT STRUCTURE AND FUNCTIONS}

An increasingly used approach to manage rivers and other ecosystems is to manipulate the physical and chemical features of the habitat. If done effectively, this stratcgy will cncourage the growth of desireable species. A similar approach can be taken with the developing GIT.

The structural and functional features of the GIT is immature when most species are born or hatched and become dependent on exogenous foods. Adult characteristics are usually not acquired until animals wean to the natural adult diet. Although the specific patterns of changes are determined by genetic factors, dictary inputs can alter the timing of development. This possibility is of particular importance in production settings when animals are weaned early. If the GIT can be preadapted by accelerating growth and maturation, the ability of developing animals to tolerate the adult diet will be acquired at any earlier stage of development.

The perinatal period is another critical phase of GIT development and some of the most profound responses to diet composition occur at birth (Zhang et al., 1998). There has been interest in identifying biologically active substances (BAS) that will accelerate GIT growth and maturation. There are three possible sources of BAS that can influence GIT development. First, many of the BAS secreted by the GIT, which represents the single largest endocrine organ in the body, are known to 
influence intestinal structure and functions (Walsh, 1981). Interestingly, many of the BAS secreted by enteric endocrine cells can be detected in the lumen (Zabielski, 1998). Second, BAS that are secreted from other sites in the body can influence the GIT. The most extensively studied examples are the glucocorticoids and thyroid hormones. Finally, dietary inputs can provide BAS that influence GIT development.

The presence of high concentrations of BAS in colostrum and longer retention in the GIT of sucklings has stimulated interest in understanding possible regulatory roles. Although a wide diversity of BAS have been identified and quantified, there remains surprisingly little information about if and how they might regulate intestinal growth and maturation (Zabielski, 1998). Although possible regulatory roles have been reported, the results are generally not consistent among species and are often controversial. To date, the majority of research about the addition of BAS to diets has focused on how to increase digestive capacities. However, other important GIT functions, such as immunity, osmoregulation, and endocrine secretions, need to be considered.

Another important consideration is that the GIT is dependent on adequate levels of dietary nutrients and energy, including essential micronutrients, such as copper and zinc (Poulsen, 1998). This is evident from GIT atrophy during parenteral nutrition. The importance of adequate dietary nutrients and energy may be even more profound during the neonatal period when they are needed to support the rapid growth of the GIT. It is now known that dietary amino acids are of particular importance (Reeds et al., 1996), with glutamine playing a central role (Pierzynowski and Sjodin, 1998). Also to be considered are the possible BAS that are produced by hydrolysis of dietary proteins, such as casein.

The viscosity of the lumenal contents is another physical feature of the GIT environments that is known to influence GIT structure, functions and the resident bacteria (Simon, 1998). The influences of lumenal viscosity are more profound during early development (Smulikowska, 1998). Higher viscosity diets reduce nutrient availability, affect enteric immune functions (Kudoh ct al., 1998), and can have been associated with changes in the bacterial assemblage that are considered detrimental (Simon, 1998). It is possible to reduce viscosity of the lumenal contents by modifying diet formulation or by the inclusion of enzymes that hydrolzye higher viscosity components, such as non starch polysaccharides.

\section{PERSPECTIVES}

Managing development of the GIT ecosystem is a complex, multifactorial problem that can be addressed using several approaches. Before establishing management strategies additional studies will be needed to address the following questions. 
When to manage? The various GIT functions and resident bacteria undergo different patterns and trajectories of development, and there is further variation between species. As a consequence, receptivity to dietary management is likely to occur at different stages of development. Exemplary of this is the limited and species-specific period for acquisition of passive immunity by neonates (reviewed by Grongnet and Duvaux-Ponter, 1998). There is a need to identify "windows of opportunity" during which the GIT is responsive to dietary management.

What to manage? The specific GIT features that are to be managed to optimize growth and health must be identified. The well known impact of the GIT bacteria and health of the host has stimulated interest in developing approaches that will promote the growth of bacterial species perceived as beneficial and/or reducing the densities of groups considered to be detrimental. Before this, though, it will be necessary to understand how the assemblages of GIT bacteria differ between species, individuals, and ages, and learn which bacteria are beneficial and those that are detrimental.

Other GIT features that may be amenable to dietary management include GIT digestive, osmoregulatory, endocrine, and immune functions. However, and as cmphasized by Kelly (1998), the interactions between GIT characteristics, the resident bacteria, and host health are complex and not well understood. Modifying one or more GIT characteristic is likely to affect others, and these interactions and their impacts need to be defined.

How to manage? There is continuing debate about the most effective strategy for managing ecosystems; by directly influencing the biota (species management), or indirectly by alterring the abiotic characteristics (habitat management). There are similar considerations concerning management of the developing GIT. Should we directly influence the species composition of the microbiota, or should we alter the physical and chemical characteristics of the habitats in order to select for a specific assemblage of bacteria?

Where should management be targetted? Most investigators examine age and diet influences in a single region of the GIT. There is a need to expand our studies to include the different habitats present in the GIT. For example, bacteriologists are often restricted to using stool samples to detect dietary influences. However, it is likely that the effects of diet on the GIT bacteria occur more proximally (McBain and MacFarlane, 1997). Furthermore, bacteria adherent to the mucosa are thought to exert more influences on the GIT than those in the lumen (Poxton et al., 1997). A better understanding of age and diet responses in the different regions of the GIT will greatly enhance our ability to devclop effective dietary strategies that will optimize GIT development, and thereby improve growth and survival of young animals. 


\section{REFERENCES}

Balmer S.E., Scott P.H., Wharton B.A., 1989. Diet and faecal flora in the newborn: lactoferrin. Arch. Dis. Child. 64, 1685-1690

Bernet M.-F., Brassart D., Neeser J.-R., Servin A.L., 1993. Adhesion of human bifidobacteria strains to culture human intestinal epithelial cells and inhibition of enteropathogen-cell interactions. Appl. Environ. Microbiol. 59, 4121-4128

Buddington R.K., 1994. Nutrition and ontogenetic development of the intestine. Can. J. Physiol. Pharmacol. 72, 25I-259

Buddington R.K., Diamond, J.D., 1989. Ontogenetic development of intestinal nutrient transporters. Ann. Rev. Physiol. 5I, 601-619

Buddington R.K., Williams C.H., Chen S.-C., Witherly S.A., 1996. Dietary supplement of neosugar alters the fecal flora and decreases activities of some reductive enzymes in human subjects. Amer. J. Clin. Nutr. 63, 709-716

Collins M.D., Gibson G.R., 1998. Nutritional modulation of microbial ecology. Amer. J. Clin. Nutr. (in press)

Conway PL., 1996. Development of intestinal microbiota. In: R.I. Mackie, B.A. Whyte, R.E. Isaacson (Editors). Gastrointestinal microbes and host interactions. Chapman and Hall, pp. 3-38

Gibson G.R., Roberfroid M.B., 1995. Dietary modulation of the human colonic microbiota: Introducing the concept of prebiotics. J. Nutr. 125, 1401-1412

Goldhill J.M., Rose K., Percy W.H., 1995. Effects of antibiotics on epithelial ion transport in the rabbit distal colon in vitro. J. Pharm. Pharmacol. 48, 651-656

Gothefors L., 1989. Effect of diet on intestinal flora. Acta Paediat. Scand., Suppl. 351, 118-121

Grongnet J.-F., Duvaux-Ponter C., 1998. Acquisition of passive immunity in domestic ungulates. J. Anim. Feed Sci. 7, Suppl. 1, 93-114

Hentges D.J., Marsh W.W., Petschow B.W., Thal W.R., Carter M.K., 1992. Influence of infant diets on the ecology of the intestinal tract of human flora-asociated mice. J. Pediat. Gastroenterol. Nutr. 14, 146-152

Howard M.D., Gordon D.T., Garleb K.A., Kerley M.S., 1995. Dietary fructooligosaccharide, xylooligosaccharide, and gum arabic have variable effects on cecal and colonic microbiota and epithelial cell proliferation in mice and rats. J. Nutr. 125, 2604-2609

Jensen B.B., 1998. The impact of feed additives on the microbial ecology of the gut in young pigs. J. Anim. Feed Sci. 7, Suppl, 1, 45-64

Kelly D., 1998. Probiotics in young and newborn animals. J. Anim. Feed Sci. 7, Suppl. 1, 15-23

Kudoh K., Shimizu J., Wada M., Takita T., Kankey., Innami S., 1998. Effect of indigestible saccarides on $\mathrm{B}$ lymphocyte response of intestinal mucosa and cecal fermentation in rats. J. Nutr. Sci. Vitaminol. 44, 103-112

McBain A.J., MacFarlane G.T., 1997. Investigations of bifidobacterial ecology and oligosaccharide metabolism in a three-stage compound continuous culture system. Scand. J. Gastroenterol. 32 Suppl. 222, 32-40

Newburg D.S., 1997. Do the binding properties of oligosaccharides in milk protect human infants from gastrointestinal bacteria? J. Nutr. 127, 980S-984S

Oli M.W., Petschow B.W., Buddington R.K., 1998. Evaluation of fructooligosaccharide supplementation of oral electrolyte solutions for treatment of diarrhea. Recovery of the intestinal bacteria. Dig. Disest. Sci. 43, 138-147

Oyofo B.A., DeLoach J.R. Corrier D.E., Norman J.O., Ziprin R.L., Mollenhauer H.H., 1989. Prevention of Salmonella typhimurium colonization of broilers with D-mannose. Poultry Sci. 68 , 1357-1360 
Oyofo B.A., Droleskey R.E., Norman J.O., Mollenhauer H.H., Ziprin R.L., Corrier D.E., DeLoach J.R., 1989. Inhibition by mannose of in vitro colonization of chicken small intestine by Salmonella typhimurium. Poulty Sci. $68,1351-1356$

Pierzynowski S.G., Sjodin A., 1998. Perspectives of glutamine and its derivatives as feed additives for farm animals. J. Anim. Feed Sci. 7, Suppl. 1, 79-91

Pierzynowski S.G., Westrøm B.R., Svendsen J., Svendsen L., Karlsson B.W., 1995. Development and regulation of porcine pancreatic function. Int. J. Pancreatol. 18, 81-94

Piva A., 1998. Non-conventional feed additives. J. Anim. Feed Sci. 7, Suppl. 1, 143-154

Poulsen H.D., 1998. Zinc and copper as feed additives, growth factors or unwanted environmental factors. J. Anim. Feed Sci. 7, Suppl. I, 135-142

Poxton I.R., Brown P., Sawyer A., Ferguson A., 1997. Mucosa-associated bacterial flora of the human colon. J. Med. Microbiol. 46, 85-91

Reeds P.J., Burrin D.G., Jahoor F., Wykes L., Henry J., Frazer E.H., 1996. Enteral glutamate is almost completely metabolized in first pass by the gastrointestinal tract of infant pigs. Amer. J. Physiol. 270, E413-E418

Roth F.X., Kirchgessner M., 1998. Organic acids as feed additives for young pigs: Nutritional and gastrointestinal effects. J. Anim. Feed Sci. 7, Suppl. 1, 25-33

Russell J.B., Diez-Gonzalez F., 1998. The effects of fermentation acids on bacterial growth. Advan. Microbial Physiol. 39, 205-234.

Simon O., 1998. The mode of action of NSP hydrolyzing enzymes in the gastrointestinal tract. $\mathbf{J}$. Anim. Feed Sci. 7, Suppl. 1, 115-123

Sangild P.T., Cranwell P.D., Hilsted L., 1992. Ontogeny of gastric function in the pig: Acid secretion and the synthesis and secretion of gastrin. Biol. Neonate 62, 363-372

Smulikowska S., 1998. Relationship between the stage of digestive tract development in chicks and the effect of viscosity-reducing enzymes on fat digestion. J. Anim. Feed Sci. 7, Suppl. 1, $125-134$

Stevens C.E., 1988. Comparative Physiology of the Vertebrate Digestive System. Cambridge University Press. Cambridge, UK

Sunvold G.D., Fahey G.C., Merchen N.R., Reinhart G.A., 1995. In vitro fermentation of selected fibrous substrates by dog and cat fecal inoculum: Influence of diet composition on substrate organic matter disappearance and short-chain fatty acid production. J. Anim. Sci. 73, $1110-1122$

Taljanski-Zygmunt W., Grzesiuk E., Pierzynowski S., 1998. Is the use of antimicrobial drugs in agriculture risky for human health? J. Anim. Feed Sci. 7, Suppl. 1, 289-295

Walsh J.H., 1981. Gastrointestinal hormones and peptides. In: L.R. Johnson (Editor). Physiology of the gastrointestinal tract. Raven Press, New York, pp. 59-144

Wegener H.C., Aarestrup F.M., Jensen L.B., Hammerum A.M., Bager F., 1998. The association between the use of antimicrobial growth promoters and development of resistance in pathogenic bacteria towards growth promoting and therapeutic antimicrobials. J. Anim. Feed Sci. 7, Suppl. $1,7-14$

Zabielski R., 1998. Regulatory peptides in milk, food, and in the gastrointestinal lumen of young animals and children. J. Anim. Feed Sci. 7, Suppl. 1, 65-78

Zhang H., Malo, C., Boyle C.R., Buddington R.K., 1998. Diet influences development of the pig (Sus scrofa) intestine during the first 6 hours after birth. J. Nutr. 128 (in press) 